\title{
The prognostic impact of circulating homeobox A9 methylated DNA in advanced non-small cell lung cancer
}

\author{
Sara Witting Christensen Wen ${ }^{1,2} \wedge$, Rikke Fredslund Andersen ${ }^{3} \wedge$, Torben Frøstrup Hansen ${ }^{1,2}$, \\ Christa Haugaard Nyhus ${ }^{1}$, Henrik Hager ${ }^{2,4}$, Ole Hilberg ${ }^{2,5}$, Anders Jakobsen ${ }^{1,2} \wedge$
}

${ }^{1}$ Department of Oncology, Vejle Hospital, University Hospital of Southern Denmark, Beriderbakken 4, 7100 Vejle, Denmark; ${ }^{2}$ Institute of Regional Health Research, University of Southern Denmark, 5230 Odense, Denmark; ${ }^{3}$ Department of Clinical Biochemistry, Vejle Hospital, University Hospital of Southern Denmark, Beriderbakken 4, 7100 Vejle, Denmark; ${ }^{4}$ Department of Pathology, Vejle Hospital, University Hospital of Southern Denmark, Beriderbakken 4, 7100 Vejle, Denmark; ${ }^{5}$ Department of Medicine, Vejle Hospital, University Hospital of Southern Denmark, Beriderbakken 4, 7100 Vejle, Denmark

Contributions: (I) Conception and design: TF Hansen, A Jakobsen; (II) Administrative support: TF Hansen, O Hilberg, A Jakobsen; (III) Provision of study materials or patients: CH Nyhus, H Hager; (IV) Collection and assembly of data: RF Andersen, SWC Wen; (V) Data analysis and interpretation: SWC Wen, TF Hansen, O Hilberg, A Jakobsen; (VI) Manuscript writing: All authors; (VII) Final approval of manuscript: All authors. Correspondence to: Sara Witting Christensen Wen. Department of Oncology, Vejle Hospital, University Hospital of Southern Denmark, Beriderbakken 4,7100 Vejle, Denmark. Email: sara.witting.christensen.wen@rsyd.dk.

Background: The homeobox A9 gene encodes a transcription factor, and aberrantly methylated homeobox A9 in the circulation has been suggested as a prognostic marker in early stage non-small cell lung cancer (NSCLC). The aim of the present study was to investigate the prognostic impact of methylated homeobox A9 in plasma from patients with advanced NSCLC.

Methods: Blood samples were prospectively collected from patients with NSCLC stage III and IV receiving standard first line chemotherapy. Sampling took place before treatment initiation and subsequently before each treatment cycle. Plasma was stored at $-80^{\circ} \mathrm{C}$ until analysis. DNA was extracted, and following bisulfite conversion methylated homeobox A9 was analyzed by methylation specific droplet digital polymerase chain reaction. Detection of methylated homeobox A9 was assessed as a binary variable. The primary endpoint was overall survival (OS).

Results: A total of 231 patients were included. At baseline methylated homeobox A9 was detected in $78.5 \%$ of the patients with a clear correlation to survival. The median OS for patients with and without detectable methylated homeobox A9 was 7.4 and 11.1 months, respectively [hazard ratio (HR) 1.79, 95\% confidence interval (CI): 1.35-2.38, $\mathrm{P}<0.001]$. The difference increased after the first cycle of treatment. At this time point the median OS was 6.2 and 15.6 months for patients with and without detectable methylated homeobox A9, respectively (HR 2.07, 95\% CI: 1.58-2.73, $\mathrm{P}<0.001)$. The independent prognostic impact of detectable methylated homeobox A9 after one treatment cycle assessed by multiple Cox regression including known prognostic factors resulted in a HR of 3.79 (2.19-6.54, P<0.001) compared to undetectable methylated homeobox A9.

Conclusions: Measurable methylated homeobox A9 after the first treatment cycle may serve as a valuable prognostic marker in patients with advanced NSCLC. Routine clinical application with treatment reconsideration calls for further studies, preferably in prospective clinical trials.

Keywords: Circulating tumor DNA; methylation; HOXA9; biomarkers; non-small cell lung cancer (NSCLC)

Submitted Jul 07, 2020. Accepted for publication Dec 23, 2020.

doi: $10.21037 /$ tlcr-20-826

View this article at: http://dx.doi.org/10.21037/tlcr-20-826

\footnotetext{
^ ORCID: Sara Witting Christensen Wen, 0000-0003-3755-0922; Rikke Fredslund Andersen, 0000-0002-5894-7745; Torben Frøstrup Hansen, 0000-0001-7476-671X; Anders Jakobsen, 0000-0003-2110-2615.
} 


\section{Introduction}

Lung cancer is estimated to have the highest cancer incidence and mortality worldwide (1). Non-small cell lung cancer (NSCLC) accounts for more than $80 \%$ of all cases. Almost half the Danish patients have metastases at the time of diagnosis, and data from England and the USA show that more than $70 \%$ are diagnosed at stage III or IV (2-4). Ageadjusted 5 -year survival rates for lung cancer stage III and IV are $9 \%$ and $3 \%$, respectively, in the Danish population (2) with similar rates in both England and the USA $(4,5)$. Overall survival (OS) has generally improved over the past years, but there is no improvement for stages III and IV in the Danish population (2) despite introduction of new treatment options.

Patients with incurable NSCLC are eligible for systemic palliative treatment if they have an Eastern Cooperative Oncology Group performance status (PS) of 0-2 (6). If there are no actionable oncogenic driver mutations and the expression level of Programmed death ligand 1 (PD-L1) is $<50 \%$, the choice is usually a double-agent chemotherapy regimen (6). A meta-analysis of 16 randomized, controlled trials showed an increase in 1-year OS from $20 \%$ to $29 \%$ when comparing chemotherapy with best supportive care (7). This translates to an increase in median OS from 4.5 to 6 months.

One of the most well-established prognostic factors in NSCLC is the tumor-node-metastasis (TNM) classification (8). However, the group of patients with advanced or locally advanced disease is large, and prognostic biomarkers are needed for further risk stratification. Liquid biopsy, a term established in recent years, has been suggested as a method for obtaining tumor material through body fluids such as blood or sputum (9-11). One way to analyze a liquid biopsy is detection of circulating tumor DNA (ctDNA) (12). Aberrant methylation patterns can be detected in most malignant tumor cells, and methylated ctDNA has been suggested for both diagnostic and prognostic purposes in patients with lung cancer (13-17). A wide range of genes have been investigated with different results, but very few assays have been clinically validated (12).

The homeobox A9 gene (HOXA9) is located on chromosome 7. It encodes a DNA-binding transcription factor involved in regulating gene expression, but its specific function is unknown. HOXA9 has been suggested as a target for microRNA-196b, with HOXA9 suppression resulting in increased cell invasiveness in vitro (18). Another study showed that HOXA9 was involved in cell migration but not proliferation in vitro (19). Methylated HOXA9 (metHOXA9) in blood and other body fluids has been suggested as a diagnostic biomarker for NSCLC (13-15,20). A few studies have investigated metHOXA9 as a prognostic marker in early stage NSCLC with promising results $(13,16,21)$. However, little is known about the methylation pattern of HOXA9 in advanced NSCLC and the prognostic information it may hold.

The aim of the present study was to investigate the prognostic impact of metHOXA9 in plasma from patients with advanced NSCLC cancer receiving standard first line treatment. We present the following article in accordance with the REMARK reporting checklist (22) (available at http://dx.doi.org/10.21037/tlcr-20-826).

\section{Methods}

\section{Study population}

The present study is a retrospective analysis of prospectively collected plasma samples from a cohort of lung cancer patients enrolled from June 2011 to September 2015 at the Department of Oncology, Vejle Hospital, Denmark. Inclusion criteria comprised age $>18$ years, histologically confirmed diagnosis of NSCLC, disease stage III or IV, PS $\leq 2$, no previous chemotherapy, and eligibility for standard first line treatment. The exclusion criteria were other malignant disease within five years prior to study enrolment except non-melanoma skin cancer, experimental treatment within 30 days prior to study enrolment, and planned radiotherapy against target lesions.

The study was conducted in accordance with the Declaration of Helsinki and the Harmonized Tripartite Guideline for Good Clinical Practice from the International Conference on Harmonization. The study was reviewed and approved by the Regional Committee on Health Research Ethics for Southern Denmark (S-20110005) and performed in accordance with Danish data protection legislation. All enrolled patients gave written, informed consent.

\section{Response evaluation}

All patients were treated and followed according to national guidelines. Treatment effect was evaluated by computed tomography (CT) scans of the chest and abdomen, and images were assessed according to the RECIST 1.1 criteria (23) by experienced radiologists. 
If no measurable target lesions were present, response status was based on non-target lesions and appearance of new malignant lesions. Treatment effect was evaluated after every two cycles or earlier as needed. Patients were censored in the event of radiation therapy against target lesions; the censor date was the date of the last evaluable CT scan. Radiologists were blinded to the biomarker status of the patients.

\section{Specimen sampling and storage}

Blood was drawn before initiation of first line medical oncology treatment (baseline), before each subsequent treatment cycle, and at confirmed disease progression. Two $9 \mathrm{ml}$ peripheral blood samples were collected into EDTAcontaining tubes and centrifuged at 2,000 $\mathrm{g}$ for 10 minutes. Plasma was stored at $-80{ }^{\circ} \mathrm{C}$ until analysis. The samples were analyzed in the period from 2017 to 2019 resulting in storage time ranging from three to eight years.

\section{Analysis of metHOXA9}

Plasma was thawed and upon reaching room temperature centrifuged at 10,000 $\mathrm{g}$ for 10 minutes. Cell free DNA was extracted from $4 \mathrm{~mL}$ plasma as previously reported (24) using the DSP Circulating DNA kit (Qiagen, Hilden, Germany) according to the manufacturer's instructions. The purified DNA was eluted in $60 \mu \mathrm{L}$ and diluted with water to a total volume of $400 \mu \mathrm{L}$. The HOXA9 methylation specific analysis was previously published by our group (25). Briefly, DNA extraction was assessed by spike-in of an internal control [CPP1, a gene from soybean (26)]. The $\beta 2$ microglobulin gene was used as a surrogate for the total amount of cell free DNA. Both CPP1 and 2 microglobulin were analyzed in triplet by quantitative PCR on $3 \mu \mathrm{L}$ of the diluted, purified DNA. The purified DNA was concentrated to a final volume of $20 \mu \mathrm{L}$. All of the purified DNA was bisulfite converted using the EZ DNA Methylation-Lightning Kit (Zymo Research, Irvine, California, USA) as recommended by the manufacturer. The converted DNA was analyzed using an in-house, HOXA9 methylation specific, droplet digital polymerase chain reaction (ddPCR) assay $(24,25)$ with albumin as reference gene (27). The primer and probe sequences as well as the PCR settings are available in Appendix 1 online. Water served as negative control, a pool of lymphocyte DNA from healthy donors $(20 \mu \mathrm{L}$ corresponding to approximately $20 \mathrm{ng}$ ) as non-cancer control, and Universal
Methylated DNA Standard (Zymo Research) as positive control. See Appendix 1 for a detailed description of the positive control. The controls were bisulfite converted and analyzed by ddPCR in parallel with the patient samples. Droplets were generated on a QX200 Automated Droplet Generator (Bio-Rad, Hercules, California, USA). Results were read on a QX100 Droplet Digital Reader (Bio-Rad). Data were exported from QuantaSoft ${ }^{\mathrm{TM}}$ (Bio-Rad) as a percentage of metHOXA9 \{calculated as [metHOXA9 copies/albumin copies] $\times 100\}$ including a $95 \%$ confidence interval (CI) derived from a Poisson distribution (28). If the CI included 0, metHOXA9 was considered undetectable in the sample. Analysis of metHOXA9 was performed blinded to the clinical endpoints.

\section{Analysis of lactate dehydrogenase $(\mathrm{LDH})$}

LDH was measured as part of the standard blood work before treatment initiation. Peripheral blood was drawn into a $4 \mathrm{~mL}$ tube containing lithium-heparin. Plasma was kept at room temperature until analysis. $\mathrm{LDH}$ was analyzed on a Roche/Hitachi Cobas 8000 c702 using a UV assay measuring photometrical increase in the absorbance. The results were reported as units/liter (U/L).

\section{Endpoints}

The primary endpoint was OS defined as the number of months from blood sampling to death of any cause. The secondary endpoint was progression free survival (PFS) defined as the number of months from blood sampling to disease progression as defined by the protocol (see 2.2. Response evaluation) or death of any cause. Patients who did not experience an event were censored at the time of data workup (1 July 2019).

\section{Statistical analyses}

Categorical variables are presented as fractions (percentages) and continuous variables as medians (interquartile range, IQR). Comparisons between groups were made with Wilcoxon rank-sum test for numeric variables and Fisher's Exact test or Pearson's Chi-Square test for categorical variables. MetHOXA9 was assessed both as a continuous (percentage) and binary (detectable or undetectable) variable. Changes in the levels of metHOXA9 were compared with Wilcoxon signed-rank test. The correlation between metHOXA9 and LDH was analyzed 


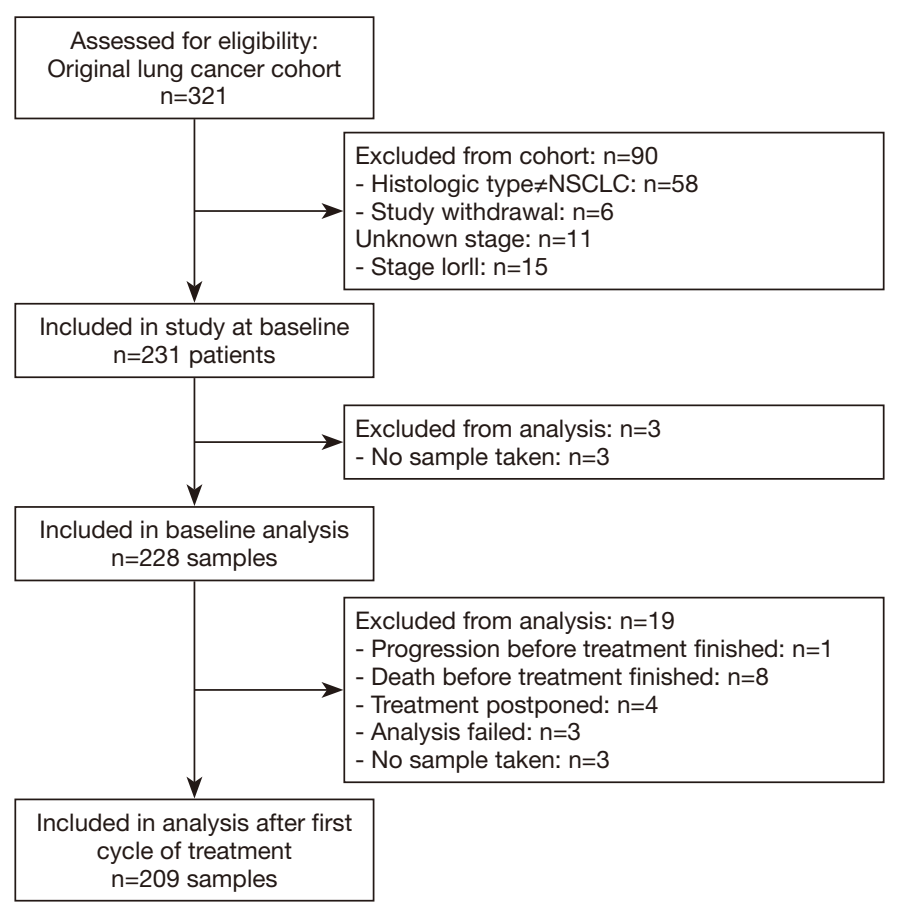

Figure 1 Flowchart illustrating the selection of patients from the original lung cancer cohort and reasons for exclusion from analysis after one cycle of treatment.

with Spearman Rank Correlation. Associations between metHOXA9 and endpoints were investigated using Kaplan-Meier curves and Log rank tests, with hazard ratio (HR) calculated by simple Cox regression. Independent prognostic impact was tested in multiple Cox regression analysis. The Cox regression model was constructed by the backward selection method including potentially prognostic factors such as sex, age, histologic cancer type, etc. Please refer to Appendix 1 online for a more detailed description of the development of the multiple Cox regression model. The model was assessed for violation of the proportional hazards assumption. All tests were two-sided with $\mathrm{P}$ values $<0.05$ considered significant. All analyses except Cox regression were performed using NCSS (NCSS, Utah, USA). Cox regression modelling was performed using STATA 16IC (StataCorp LLC, Texas, USA).

\section{Results}

\section{Patient characteristics}

A total of 231 patients met the inclusion criteria out of 321 patients from the original cohort (Figure 1). At baseline 228 samples were available, and after the first cycle of treatment and at disease progression 209 and 134 samples, respectively, were included for analysis. Of the 231 patients included, 194 had disease progression (84\%) and 226 had died (97.8\%). Only one patient neither experienced progression nor death during the follow-up period. Median follow-up time was not calculated since the vast majority of patients experienced an event.

Patient characteristics are shown in Table 1. There was no significant difference between the methylation groups regarding sex, histology, stage, smoking status, and treatment. The group with no detectable metHOXA9 at baseline was characterized by significantly older patients and lower LDH compared to the group with detectable metHOXA9.

\section{Dynamics of metHOXA9 in advanced NSCLC}

The median level of metHOXA9 at baseline was $1.65 \%$ (IQR: $0.30-5.85 \%$ ) with $21.5 \%$ of the patients having undetectable metHOXA9. After the first treatment cycle the median metHOXA9 was $0.53 \%$ (IQR: $0-2.80 \%$ ) and the percentage of patients with undetectable metHOXA9 had significantly increased to $34.4 \%(\mathrm{P}<0.001)$. At progression, the median level of metHOXA9 was $1.49 \%$ (IQR: 0.20 
Table 1 Patient characteristics of the whole cohort and as divided into subgroups by metHOXA9 status at baseline

\begin{tabular}{|c|c|c|c|c|}
\hline Patient characteristics & Total, $\mathrm{n}=231$ & Baseline metHOXA9 $=0, n=49$ & Baseline metHOXA9 >0, $n=179$ & $P$ value \\
\hline Age, years & $67[61-74]$ & $71[64-75]$ & 66 [60-73] & $0.017^{\star}$ \\
\hline Histologic type & & & & 0.064 \\
\hline Adenocarcinoma & $180(78 \%)$ & $43 / 88 \%)$ & $134(75 \%)$ & \\
\hline Other $^{\dagger}$ & $18(8 \%)$ & $4(8 \%)$ & $14(8 \%)$ & \\
\hline Stage & & & & 1.000 \\
\hline III & $32(14 \%)$ & $7(14 \%)$ & $25(14 \%)$ & \\
\hline IV & $199(86 \%)$ & $42(86 \%)$ & $154(86 \%)$ & \\
\hline Other $^{\ddagger}$ & $29(13 \%)$ & $6(12 \%)$ & $22(12 \%)$ & \\
\hline Smoking status & & & & 0.241 \\
\hline Never & $16(7 \%)$ & $6(12 \%)$ & $10(6 \%)$ & \\
\hline Previous & $136(59 \%)$ & $26(53 \%)$ & $109(61 \%)$ & \\
\hline Active & $74(32 \%)$ & $16(33 \%)$ & $56(31 \%)$ & \\
\hline Unknown ${ }^{\S}$ & $5(2 \%)$ & $1(2 \%)$ & $4(2 \%)$ & \\
\hline LDH baseline, units/L & $202[171-254]$ & $175.5[160-224]$ & 205 [176-275] & $<0.001^{*}$ \\
\hline LDH baseline, $\geq 250 \mathrm{U} / \mathrm{L}$ & $60 / 215(28 \%)$ & $4 / 46(2 \%)$ & $56 / 169(26 \%)$ & $<0.001^{\star}$ \\
\hline
\end{tabular}

Results are presented as a median (IQR) for numeric variables and a fraction (percentage) for categorical variables. Differences between groups were tested by Wilcoxon rank-sum test for numeric variables and by Fisher's exact test or Pearson's Chi square test for categorical variables as appropriate. * , statistically significant difference between groups; ${ }^{\dagger}$, category covers poorly differentiated non-small cell carcinoma and tumors with mixed histology treated as NSCLC; ${ }^{\ddagger}$, category covers vinorelbine monotherapy and tyrosine kinase inhibitors; ${ }^{\S}$, treated as missing values in analyses. LDH, lactate dehydrogenase; NSCLC, non-small cell lung cancer.

$7.06 \%$ ) with $17.9 \%$ of the patients having undetectable metHOXA9. The level of metHOXA9 increased in the three samples before disease progression. The median values were $0 \%(0-0.88 \%), 0.19 \%(0-0.90 \%)$, and $0.44 \%$ $(0-1.88 \%)$, respectively. This increase, however, was not statistically significant (sample 3 compared to sample 5, $\mathrm{P}=0.054$, Figure 2).

\section{LDH and survival}

There was a weak correlation between $\mathrm{LDH}$ and metHOXA9 ( $\mathrm{r}=0.31,95 \%$ CI: $0.18-0.43, \mathrm{P}<0.001$ ). There was no significant association between PFS and elevated $\mathrm{LDH}(\mathrm{LDH} \geq 250 \mathrm{U} / \mathrm{L}, \mathrm{P}=0.150, \mathrm{n}=218)$. Patients with normal range $\mathrm{LDH}(<250 \mathrm{U} / \mathrm{L})$ had a median $\mathrm{OS}$ of
8.4 months while patients with elevated LDH had a median OS of 6.2 months, HR 1.40, (95\% CI: 1.01-1.93, $\mathrm{P}=0.027$, $\mathrm{n}=218$ ). Kaplan-Meier plots for LDH status and other patient characteristics can be found in Appendix 1 online.

\section{HOXA9-methylation and survival}

The status of metHOXA9 was highly associated with survival. The median PFS in patients with and without baseline detectable metHOXA9 was 4.2 and 6.0 months, respectively, HR 1.57 (95\% CI: 1.18-2.09, p=0.0041, $\mathrm{n}=228$, Figure $3 A$ ). After one cycle of treatment the median PFS was 2.7 and 5.3 months in patients with and without metHOXA9, respectively, HR 1.74 (95\% CI: 1.33-2.29, $\mathrm{P}=0.0001, \mathrm{n}=209$, Figure 3 B). This difference also applied 


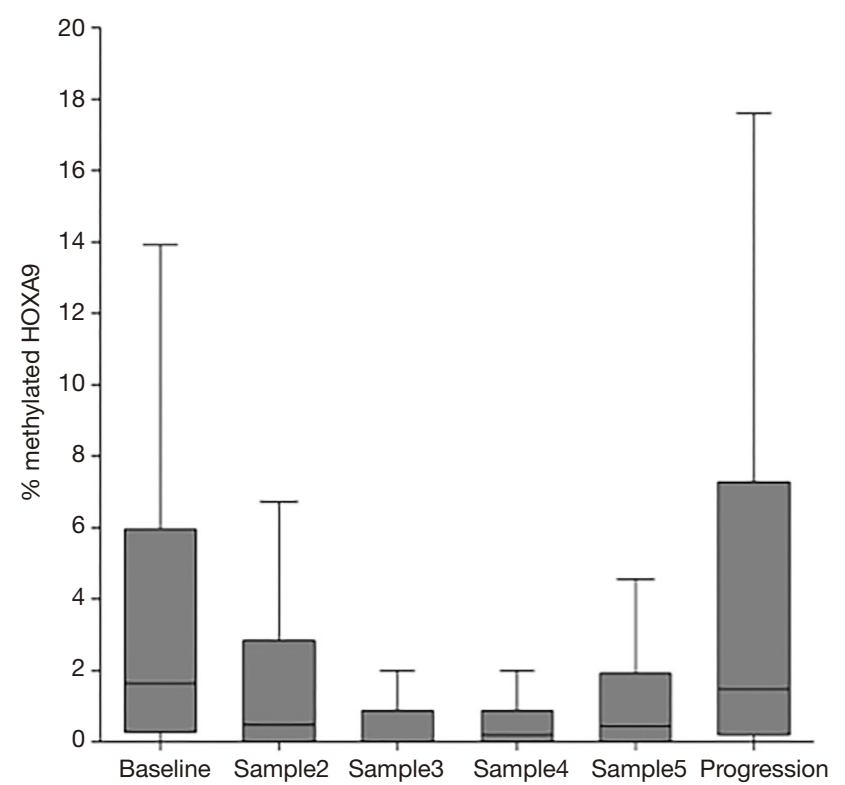

Figure 2 Box plot illustrating the dynamics of methylated HOXA9 from baseline, after the first cycle of treatment (sample 2), at the three samples before progression (samples 3-5), and after disease progression as confirmed by CT scan.
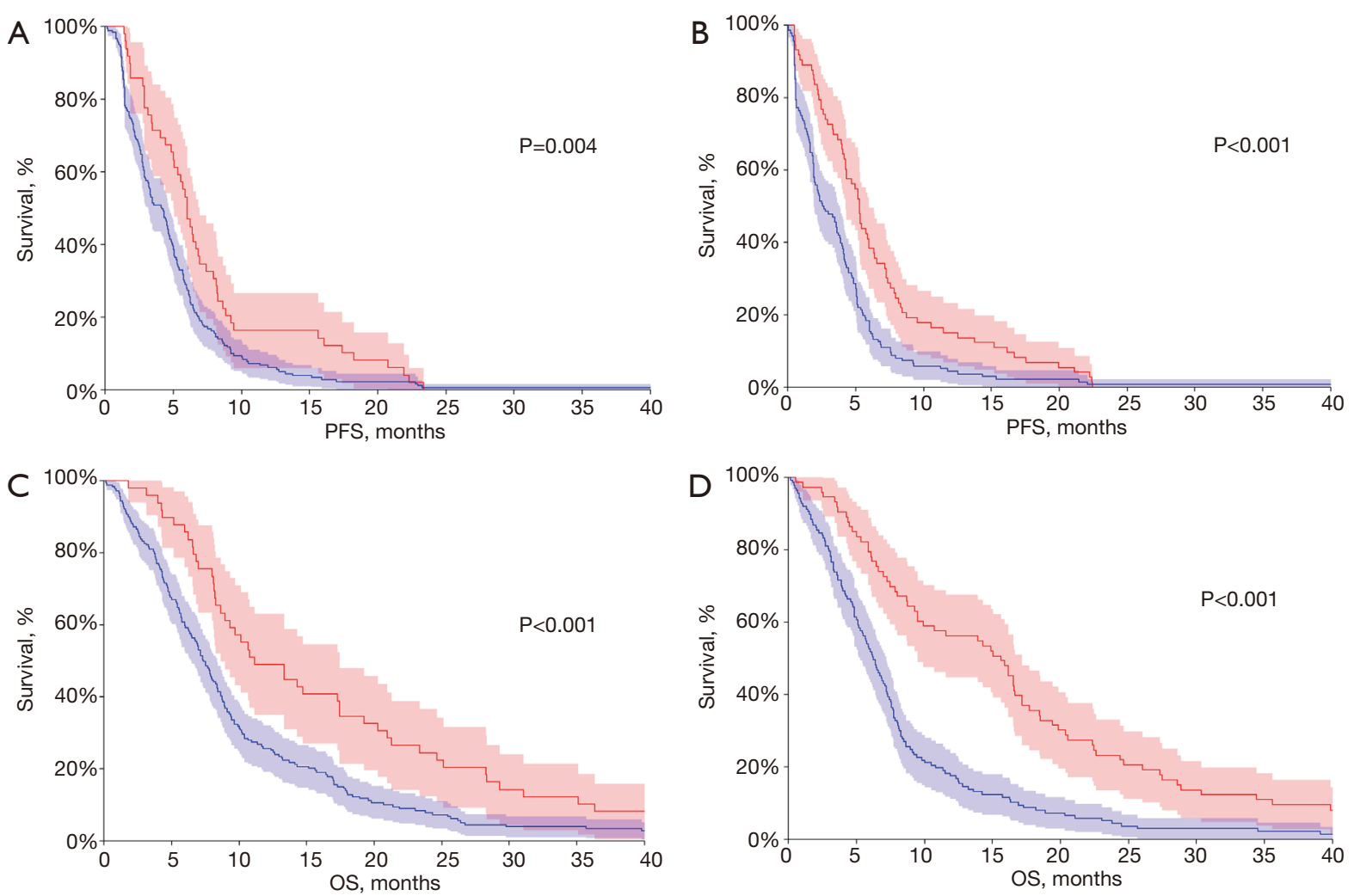

Figure 3 Prognostic impact of metHOXA9 status. Kaplan-Meier plots illustrating PFS and OS probability as a function of metHOXA9 status at baseline $(\mathrm{A}, \mathrm{C})$ and after the first treatment cycle $(\mathrm{B}, \mathrm{D})$. Red line: metHOXA9 $=0$. Blue line: metHOXA9 $>0$. Colored areas represent 95\% CIs. P values for Log rank tests are shown. PFS, progression free survival; OS, overall survival. 
Table 2 Multiple Cox regression analysis performed on 196 patients and 193 events with complete data for all covariates

\begin{tabular}{|c|c|c|c|c|}
\hline Covariate & $\mathrm{HR}$ & $95 \% \mathrm{Cl}$ (lower) & $95 \% \mathrm{Cl}$ (upper) & $P$ value \\
\hline Undetectable (reference) & 1 & & & \\
\hline Detectable & 3.788 & 2.192 & 6.544 & $<0.001^{*}$ \\
\hline \multicolumn{5}{|l|}{ Sex } \\
\hline Male & 1.299 & 0.970 & 1.740 & 0.079 \\
\hline \multicolumn{5}{|l|}{ Treatment } \\
\hline Platinum and vinorelbine (reference) & 1 & & & \\
\hline Other than platinum and vinorelbine & 0.637 & 0.405 & 1.002 & 0.051 \\
\hline
\end{tabular}

Table presents hazard ratios (HR), 95\% Cls, and P values. *, statistically significant covariates. $\mathrm{Cl}$, confidence interval.

to OS with a median of 7.4 and 11.1 months, respectively, for the patients with and without detectable metHOXA9, HR 1.79 (95\% CI: $1.35-2.38, \mathrm{P}=0.0003, \mathrm{n}=228$, Figure 3C). The prognostic impact became clearer after the first cycle of treatment with an OS of 6.2 and 15.6 months for patients with and without detectable metHOXA9, respectively, HR 2.07 (95\% CI: 1.58-2.73, $\mathrm{P}<0.0001, \mathrm{n}=209$, Figure 3D).

\section{Independent prognostic impact of metHOXA9}

The independent prognostic impact of detectable metHOXA9 after the first cycle of treatment was assessed by multiple Cox regression. There was an independent prognostic impact of LDH but only borderline significant impact of sex and treatment (Table 2). Since the status of metHOXA9 violated the proportional hazards assumption, a time-varying coefficient (metHOXA9 status interacting with time) was introduced into the Cox model. The HR of detectable metHOXA9 is $3.79(2.19-6.54, \mathrm{P}<0.001)$ at time $=0$. The HR declines by 0.002 at each unit of time. This means that the HR would be 3.06 at time $=365$ days or 2.33 at time $=730$ days. The Cox modelling process is described in further detail in Appendix 1 online.

\section{Discussion}

This study, to the best of our knowledge, is the first to explore the prognostic impact of circulating metHOXA9 in advanced NSCLC. We show that detection of metHOXA9 at baseline is a negative prognostic factor in patients with NSCLC stage III and IV, and the impact is enhanced after the first treatment cycle.

Previous studies have shown metHOXA9 to be a promising prognostic marker. Ooki et al. (13) reported a tendency towards worse OS in patients with detectable metHOXA9. However, the cohort was rather small $(n=53)$ and metHOXA9 was detected by quantitative methylation specific PCR (Q-MSP), which is a well-established method but not as precise or accurate as ddPCR (29). Lissa et al. (30) found that metHOXA9 was independently associated with worse cancer specific survival in patients with stage I adenocarcinoma of the lung. Sandoval et al. (16) reported a significantly shorter relapse free survival for operated stage I lung cancer patients with detectable metHOXA9 in both their discovery and validation cohort. All of these studies used tumor tissue for metHOXA9 analysis in relation to prognosis, and the patients had localized disease. We have now shown that metHOXA9 is a prognostic biomarker in advanced NSCLC as well, and that it can be detected in plasma. This finding is supported by Peng et al. (31), who detected methylated ctDNA in the form of short stature homeobox 2 (SHOX2). They reported a significantly better OS in stage IV NSCLC patients with low pre-treatment and post-treatment SHOX2 levels compared to patients with higher levels.

The diagnostic sensitivity of metHOXA9 tends to be higher in tissue samples than ctDNA from blood or pleural effusion (13), but this might be countered by choosing a 
more sensitive detection method or increasing the volume of samples. We used ddPCR for ctDNA detection, which is considered a reproducible method (32), and included both internal and external controls for validation. The method is cost-efficient and relatively simple, making implementation into routine clinical practice feasible. metHOXA9 has generally shown good performance and sensitivity in previous studies $(13-15,20,33)$. Nevertheless, the assay does not have perfect sensitivity, and the patient may have a lung cancer which does not release metHOXA9. Sensitivity seems to increase with a panel of genes, though a trade-in on specificity has been observed (13). By building a gene panel analyzed with ddPCR the sensitivity may be improved and more accurate results generated.

Interestingly, we found the prognostic impact of metHOXA9 status to interact with time, meaning that it violated the proportional hazards assumption. This can be interpreted as a declining prognostic impact of metHOXA9 status; i.e., detectable metHOXA9 after the first treatment cycle has a certain impact on prognosis, but the impact gradually decreases over time. Accounting for a timevarying coefficient can lead to more solid estimates in the Cox regression model, if it makes clinical and logical sense $(34,35)$. An even more precise prognosis estimate may be derived from real-time monitoring with serial blood sampling. This has shown clinical potential with a relevant lead time compared to standard follow-up with CT scans in the progression of colon cancer (36) and recurrence of bladder cancer (37).

We observed an independent prognostic impact of LDH in the multiple Cox regression confirming previous findings. An elevated LDH level was associated with shorter OS in a meta-analysis of 76 studies of patients with solid tumors and the same applied to their subgroup analysis of seven studies on lung cancer patients (38). Similar results were found in a recent meta-analysis of lung cancer studies (39). A study in colorectal cancer found a correlation between methylation of two genes and LDH to be a surrogate marker for cell death (40). However, this was not the case for a third gene investigated, suggesting that ctDNA can occur by other routes than cell death.

After the first cycle of treatment the fraction of patients with undetectable metHOXA9 was higher than at baseline, i.e., the treatment changed the biomarker status for some patients. The difference in OS between patients with and without detectable metHOXA9 was more than doubled after the first treatment cycle. Similar results were recently reported by our group in a study of circulating metHOXA9 in ovarian cancer, in which the most pronounced effect was observed after the third treatment cycle with an OS of 9.4 months versus 19.4 months in patients with detectable and undetectable metHOXA9, respectively (25). This implies that a change in biomarker status reflects a change of prognosis. The difference in metHOXA9 was observed after only three weeks in the present study while the first response evaluation by CT scan was performed after six to eight weeks depending on treatment regimen. The large difference in OS according to metHOXA9 after the first treatment cycle raises the question of whether treatment should be reconsidered for patients with detectable metHOXA9. There is a possibility of this marker being predictive as well as prognostic. The patients with detectable metHOXA9 after the first treatment cycle potentially do not benefit from the administered chemotherapy regimen and may be better served by moving on to second line treatment, e.g., immunotherapy. This remains to be elucidated in a prospective trial ideally randomizing patients to standardized follow-up with CT evaluation of treatment response or follow-up by metHOXA9 measurement for treatment reconsideration.

The primary limitation of the present study is its retrospective nature increasing the possibility of selection bias. However, the plasma samples were prospectively collected with the purpose of future biomarker analysis, and the translational study was pre-planned. The clinical endpoints were unknown at the time of collection and laboratory staff were blinded to clinical outcome status of the individual patient. The samples were stored for up to eight years, which is rather a long time. They were never thawed and refrozen, and we did not experience any problems with DNA yield. However, it was previously reported that the loss of cell free DNA in frozen plasma samples was about $30 \%$ per year of storage (41). Mean and median cell free DNA yield in the present study can be found in Appendix 1 online. We believe that the storage time did not affect our samples to a degree that would significantly change our results, although shorter storage time would have been preferable. While the cohort is of intermediate size, the samples were collected at a single institution with no validation cohort. These results should therefore be interpreted with caution until further validated. The cohort is Danish and thus quite uniformly Caucasian, meaning that the results should be applied to other countries with caution, as genes and methylation patterns may vary between ethnic groups. And finally, we only investigated methylation of one gene as elaborated above. 
In conclusion, the present study suggests that detectable circulating metHOXA9 is a negative prognostic factor in patients with advanced NSCLC and could be used for risk stratification after one cycle of treatment. Routine clinical application requires further investigation in prospective clinical trials.

\section{Acknowledgments}

We would like to thank Yvette Schandorf Sørensen for collecting data with great attention to detail, Pia Nielsen, Lone Hartmann Hansen, and Tina Brandt Christensen for their work in the laboratory, Marianne Skaarup for her assistance in collecting data, and Karin Larsen for her language editing assistance. An abstract with preliminary results from this study was accepted for online publication at ASCO 2018.

Funding: This work was supported by Gangstedfonden (granted to TH) and grants from the Department of Oncology, Vejle Hospital, University Hospital of Southern Denmark.

\section{Footnote}

Reporting Checklist: The authors have completed the REMARK reporting checklist. Available at http://dx.doi. org/10.21037/tlcr-20-826

Data Sharing Statement: Available at http://dx.doi. org/10.21037/tlcr-20-826

Peer Review File: Available at http://dx.doi.org/10.21037/ tlcr-20-826

Conflicts of Interest: All authors have completed the ICMJE uniform disclosure form (available at http://dx.doi. org/10.21037/tlcr-20-826). SW, TH, RA, CN, OH, and AJ report non-financial support from NKMax America, outside the submitted work. The authors have no other conflicts of interest to declare.

Ethical Statement: The authors are accountable for all aspects of the work in ensuring that questions related to the accuracy or integrity of any part of the work are appropriately investigated and resolved. The study was conducted in accordance with the Declaration of Helsinki (as revised in 2013) and the Harmonized Tripartite Guideline for Good Clinical Practice from the International
Conference on Harmonization. The study was reviewed and approved by the Regional Committee on Health Research Ethics for Southern Denmark (S-20110005) and performed in accordance with Danish data protection legislation. All enrolled patients gave written, informed consent.

Open Access Statement: This is an Open Access article distributed in accordance with the Creative Commons Attribution-NonCommercial-NoDerivs 4.0 International License (CC BY-NC-ND 4.0), which permits the noncommercial replication and distribution of the article with the strict proviso that no changes or edits are made and the original work is properly cited (including links to both the formal publication through the relevant DOI and the license). See: https://creativecommons.org/licenses/by-nc-nd/4.0/.

\section{References}

1. Bray F, Ferlay J, Soerjomataram I, et al. Global cancer statistics 2018: GLOBOCAN estimates of incidence and mortality worldwide for 36 cancers in 185 countries. CA Cancer J Clin 2018;68:394-424.

2. Jakobsen E, Rasmussen TR, Green A. Mortality and survival of lung cancer in Denmark: Results from the Danish Lung Cancer Group 2000-2012. Acta Oncol 2016;55:2-9.

3. Cancer Research UK, Lung cancer incidence statistics [Internet]. [cited $2020 \mathrm{Jul}$ 1]. Available online: https://www. cancerresearchuk.org/health-professional/cancer-statistics/ statistics-by-cancer-type/lung-cancer/incidence\#headingThree

4. Howlader N, Noone AM, Krapcho M, et al. SEER Cancer Statistics Review, 1975-2016, National Cancer Institute. Bethesda, MD. Based on November 2018 SEER data submission, posted to the SEER web site, April 2019. [Internet]. [cited $2020 \mathrm{Jul}$ 1]. Available online: https://seer. cancer.gov/csr/1975_2016/results_merged/sect_15_lung_ bronchus.pdf

5. Cancer Research UK, Lung cancer survival statistics [Internet]. [cited $2020 \mathrm{Jul} 1$ 1]. Available online: https:// www.cancerresearchuk.org/health-professional/cancerstatistics/statistics-by-cancer-type/lung-cancer/survival\#ref

6. Planchard D, Popat S, Kerr K, et al. Metastatic non-small cell lung cancer: ESMO Clinical Practice Guidelines for diagnosis, treatment and follow-up. Ann Oncol Off J Eur Soc Med Oncol 2018;29:iv192-237.

7. Non-Small Cell Lung Cancer Collaborative Group. Chemotherapy and supportive care versus supportive care 
alone for advanced non-small cell lung cancer. Cochrane Database Syst Rev 2010;(5):CD007309.

8. Chansky K, Detterbeck FC, Nicholson AG, et al. The IASLC Lung Cancer Staging Project: External Validation of the Revision of the TNM Stage Groupings in the Eighth Edition of the TNM Classification of Lung Cancer. J Thorac Oncol 2017;12:1109-21.

9. Pantel K, Alix-Panabières C. Circulating tumour cells in cancer patients: challenges and perspectives. Trends Mol Med 2010;16:398-406.

10. Mader S, Pantel K. Liquid Biopsy: Current Status and Future Perspectives. Oncol Res Treat 2017;40:404-8.

11. Revelo AE, Martin A, Velasquez R, et al. Liquid biopsy for lung cancers: an update on recent developments. Ann Transl Med 2019;7:349.

12. Merker JD, Oxnard GR, Compton C, et al. Circulating Tumor DNA Analysis in Patients With Cancer: American Society of Clinical Oncology and College of American Pathologists Joint Review. J Clin Oncol 2018;36:1631-41.

13. Ooki A, Maleki Z, Tsay JCJ, et al. A Panel of Novel Detection and Prognostic Methylated DNA Markers in Primary Non-Small Cell Lung Cancer and Serum DNA. Clin Cancer Res 2017;23:7141-52.

14. Hulbert A, Jusue-Torres I, Stark A, et al. Early Detection of Lung Cancer Using DNA Promoter Hypermethylation in Plasma and Sputum. Clin Cancer Res 2017;23:1998-2005.

15. Yang Z, Qi W, Sun L, et al. DNA methylation analysis of selected genes for the detection of early-stage lung cancer using circulating cell-free DNA. Adv Clin Exp Med 2019;28:355-60.

16. Sandoval J, Mendez-Gonzalez J, Nadal E, et al. A Prognostic DNA Methylation Signature for Stage I NonSmall-Cell Lung Cancer. J Clin Oncol 2013;31:4140-7.

17. Balgkouranidou I, Chimonidou M, Milaki G, et al. SOX17 promoter methylation in plasma circulating tumor DNA of patients with non-small cell lung cancer. Clin Chem Lab Med 2016;54:1385-93.

18. Yu SL, Lee DC, Sohn HA, et al. Homeobox A9 directly targeted by miR-196b regulates aggressiveness through nuclear Factor-kappa B activity in non-small cell lung cancer cells. Mol Carcinog 2016;55:1915-26.

19. Hwang JA, Lee BB, Kim Y, et al. HOXA9 inhibits migration of lung cancer cells and its hypermethylation is associated with recurrence in non-small cell lung cancer. Mol Carcinog 2015;54 Suppl 1:E72-80.

20. Nawaz I, Qiu X, Wu H, et al. Development of a multiplex methylation specific PCR suitable for (early) detection of non-small cell lung cancer. Epigenetics 2014;9:1138-48.

21. Robles AI, Arai E, Mathé EA, et al. An Integrated Prognostic Classifier for Stage I Lung Adenocarcinoma Based on mRNA, microRNA, and DNA Methylation Biomarkers. J Thorac Oncol 2015;10:1037-48.

22. Mallett S, Timmer A, Sauerbrei W, et al. Reporting of prognostic studies of tumour markers: a review of published articles in relation to REMARK guidelines. Br J Cancer 2010;102:173-80.

23. Eisenhauer EA, Therasse P, Bogaerts J, et al. New response evaluation criteria in solid tumours: revised RECIST guideline (version 1.1). Eur J Cancer 2009;45:228-47.

24. Thomsen CB, Andersen RF, Steffensen KD, et al. Delta tocotrienol in recurrent ovarian cancer. A phase II trial. Pharmacol Res 2019;141:392-6.

25. Rusan M, Andersen RF, Jakobsen A, et al. Circulating HOXA9-methylated tumour DNA: A novel biomarker of response to poly (ADP-ribose) polymerase inhibition in BRCA-mutated epithelial ovarian cancer. Eur J Cancer 2020;125:121-9.

26. Pallisgaard N, Spindler KLG, Andersen RF, et al. Controls to validate plasma samples for cell free DNA quantification. Clin Chim Acta 2015;446:141-6.

27. Roperch JP, Incitti R, Forbin S, et al. Aberrant methylation of NPY, PENK, and WIF1 as a promising marker for blood-based diagnosis of colorectal cancer. BMC Cancer 2013;13:566.

28. Droplet Digital PCR Applications Guide [Internet]. Bio-Rad; [cited 2019 Nov 27]. Available online: http:// www.bio-rad.com/webroot/web/pdf/lsr/literature/ Bulletin_6407.pdf

29. Wiencke JK, Bracci PM, Hsuang G, et al. A comparison of DNA methylation specific droplet digital PCR (ddPCR) and real time qPCR with flow cytometry in characterizing human $T$ cells in peripheral blood. Epigenetics 2014;9:1360-5.

30. Lissa D, Ishigame T, Noro R, et al. HOXA9 methylation and blood vessel invasion in FFPE tissues for prognostic stratification of stage I lung adenocarcinoma patients. Lung Cancer 2018;122:151-9.

31. Peng X, Liu $X, X u L$, et al. The $\operatorname{mSHOX} 2$ is capable of assessing the therapeutic effect and predicting the prognosis of stage IV lung cancer. J Thorac Dis 2019;11:2458-69.

32. Whale AS, Devonshire AS, Karlin-Neumann G, et al. International Interlaboratory Digital PCR Study Demonstrating High Reproducibility for the Measurement of a Rare Sequence Variant. Anal Chem 2017;89:1724-33. 
33. Belinsky SA, Leng S, Wu G, et al. Gene Methylation Biomarkers in Sputum and Plasma as Predictors for Lung Cancer Recurrence. Cancer Prev Res (Phila) 2017;10:635-40.

34. Zhang Z, Reinikainen J, Adeleke KA, et al. Time-varying covariates and coefficients in Cox regression models. Ann Transl Med 2018;6:121.

35. Patrick Royston DWH. Using Aalen's linear hazards model to investigate time-varying effects in the proportional hazards regression model. Stata J 2002;2:331-50.

36. Thomsen CB, Hansen TF, Andersen RF, et al. Monitoring the effect of first line treatment in RAS/RAF mutated metastatic colorectal cancer by serial analysis of tumor specific DNA in plasma. J Exp Clin Cancer Res 2018;37:55.

37. Christensen E, Birkenkamp-Demtröder K, Sethi H, et al. Early Detection of Metastatic Relapse and Monitoring of Therapeutic Efficacy by Ultra-Deep Sequencing of Plasma
Cell-Free DNA in Patients With Urothelial Bladder Carcinoma. J Clin Oncol 2019;37:1547-57.

38. Petrelli F, Cabiddu M, Coinu A, et al. Prognostic role of lactate dehydrogenase in solid tumors: a systematic review and meta-analysis of 76 studies. Acta Oncol 2015;54:961-70.

39. Deng T, Zhang J, Meng Y, et al. Higher pretreatment lactate dehydrogenase concentration predicts worse overall survival in patients with lung cancer. Medicine (Baltimore) 2018;97:e12524.

40. Philipp AB, Nagel D, Stieber P, et al. Circulating cell-free methylated DNA and lactate dehydrogenase release in colorectal cancer. BMC Cancer 2014;14:245.

41. Sozzi G, Roz L, Conte D, et al. Effects of Prolonged Storage of Whole Plasma or Isolated Plasma DNA on the Results of Circulating DNA Quantification Assays. JNCI J Natl Cancer Inst 2005;97:1848-50.

Cite this article as: Wen SWC, Andersen RF, Hansen TF, Nyhus CH, Hager H, Hilberg O, Jakobsen A. The prognostic impact of circulating homeobox A9 methylated DNA in advanced non-small cell lung cancer. Transl Lung Cancer Res 2021;10(2):855-865. doi: 10.21037/tlcr-20-826 


\section{Appendix 1}

\section{Investigators}

Sara Witting Christensen Wen ${ }^{1,2}$, MD, Rikke Fredslund Andersen ${ }^{3}$, MSc., PhD, Torben Frøstrup Hansen ${ }^{1,2}$, associate Professor, MD, PhD, Christa Haugaard Nyhus ${ }^{1}$, MD, Henrik Hager ${ }^{2,4}$ associate professor, MD, PhD, Ole Hilberg ${ }^{2,5}$, professor, MD, DMSc, Anders Jakobsen ${ }^{1,2}$, professor, MD, DMSc

${ }^{1}$ Department of Oncology, Vejle Hospital, University Hospital of Southern Denmark, Beriderbakken 4, 7100 Vejle, Denmark

${ }^{2}$ Institute of Regional Health Research, University of Southern Denmark, 5230 Odense, Denmark

${ }^{3}$ Department of Clinical Biochemistry, Vejle Hospital, University Hospital of Southern Denmark, Beriderbakken 4, 7100 Vejle, Denmark

${ }^{4}$ Department of Pathology, Vejle Hospital, University Hospital of Southern Denmark, Beriderbakken 4, 7100 Vejle, Denmark

${ }^{5}$ Department of Medicine, Vejle Hospital, University Hospital of Southern Denmark, Beriderbakken 4, 7100 Vejle, Denmark

\section{Methods}

Methylated HOXA9 was analyzed with an in-house ddPCR assay. (Primers and probe from LGC Biosearch technologies, Aarhus, Denmark).

Primer sequence

Forward

GAGTATTTCGATTTTAGTTCGTGT

Reverse

CGCGTACACTAAATTCCAC

Probe sequence

Probe

\section{FAM-TTAGTTTAAGGCGACGGTGTT-BHQ-1}

Positive control:

Universal Methylated Human DNA Standard, $2 \mu \mathrm{l}$ (DNA concentration 250 ng/ $\mu \mathrm{l}$, Zymo Research, Irvine, California, USA), healthy donor lymphocyte DNA $199 \mu \mathrm{l}$ (DNA concentration approximately $20 \mathrm{ng} / \mu \mathrm{l}$ ) and water $800 \mu \mathrm{l}$.

For each reaction, $20 \mu \mathrm{l}$ of this mixture was added and bisulfite converted in parallel with the patient samples.

PCR conditions for the methylation specific droplet digital PCR assay

\begin{tabular}{|l|c|c|}
\hline Steps & Temperature & Time \\
\hline Step 1 & $95^{\circ} \mathrm{C}$ & 10 minutes \\
\hline Step 2: 44 cycles & $95^{\circ} \mathrm{C}$ & 15 seconds \\
& $56^{\circ} \mathrm{C}$ & 1 minute \\
\hline Step 3 & $98^{\circ} \mathrm{C}$ & 10 minutes \\
\hline
\end{tabular}

Machine: Veriti Thermal Cycler (Applied Biosystems, Thermo Fisher Scientific, Foster City, California, USA).

Ramp rate: $1.5^{\circ} \mathrm{C} /$ second.

Cell free DNA yield:

We have measured total cell-free DNA by qPCR in the form of the $\beta 2$ microglobulin gene in the present patient cohort. We found a median of 2825 copies $/ \mathrm{ml}$ (mean 4920 copies $/ \mathrm{ml}$, range 279-106656 copies/ml) across the 228 baseline samples.

\section{Univariate analyses}
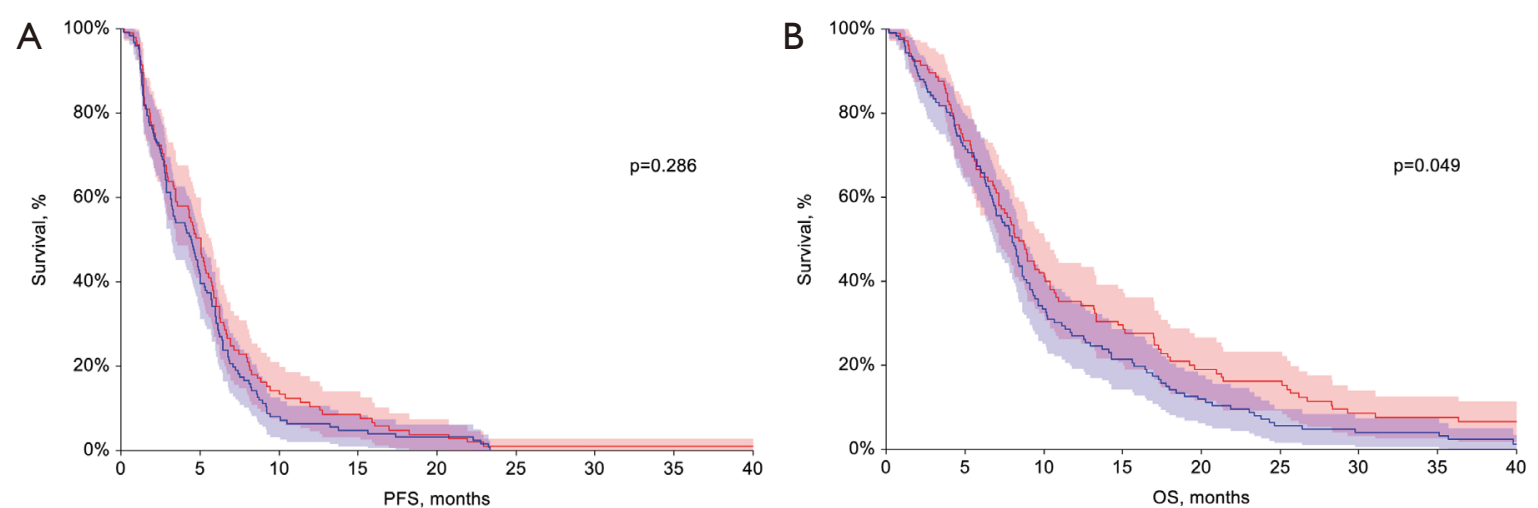

Figure S1 Prognostic impact of sex. Kaplan-Meier plots illustrating PFS and OS probability as a function of sex (A, B). Red line: Female. Blue line: Male. Colored areas represent $95 \%$ CIs. P-values for Log rank tests are shown. 

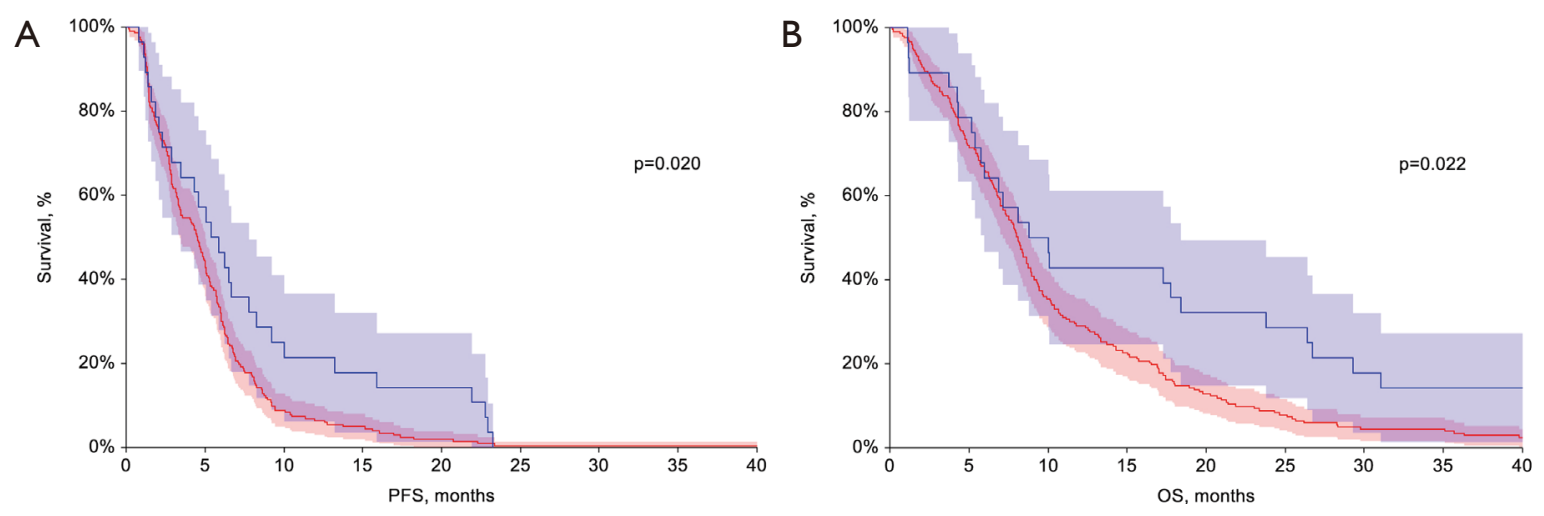

Figure S2 Prognostic impact of treatment. Kaplan-Meier plots illustrating PFS and OS probability as a function of treatment category (A, B). Red line: Platinum and vinorelbine. Blue line: Other treatments. The category 'Other' covers vinorelbine monotherapy and tyrosine kinase inhibitors. Colored areas represent 95\% CIs. P-values for Log rank tests are shown.
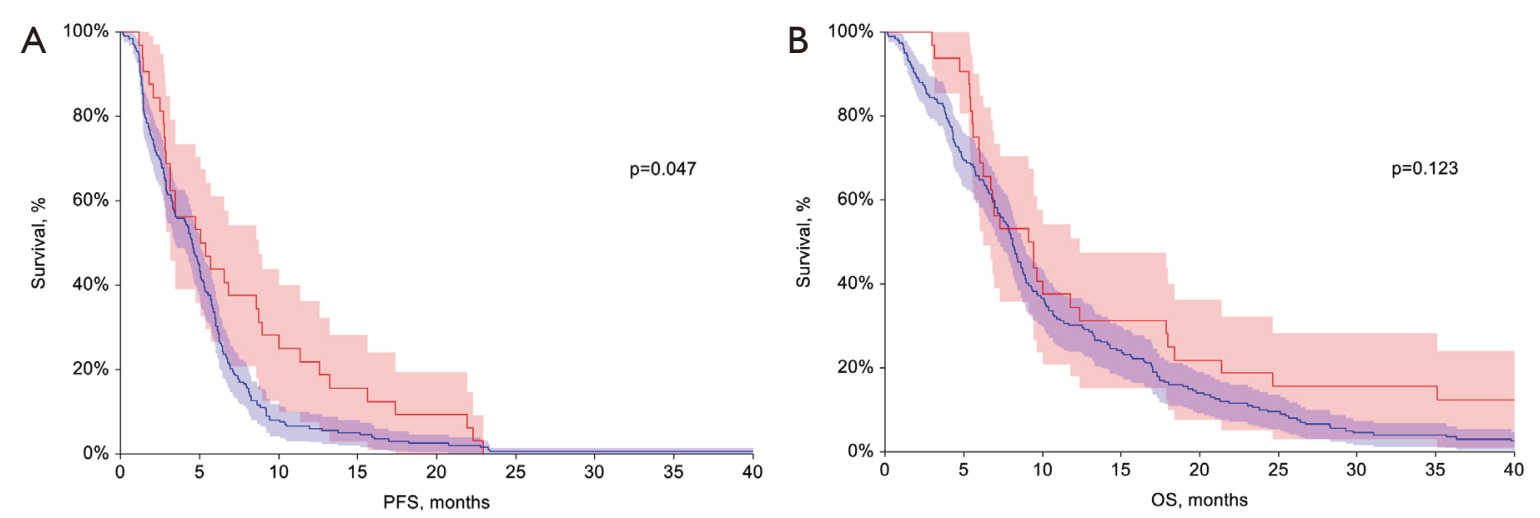

Figure S3 Prognostic impact of stage. Kaplan-Meier plots illustrating PFS and OS probability as a function of stage (A, B). Red line: Stage 3. Blue line: Stage 4. Colored areas represent 95\% CIs. P-values for Log rank tests are shown.
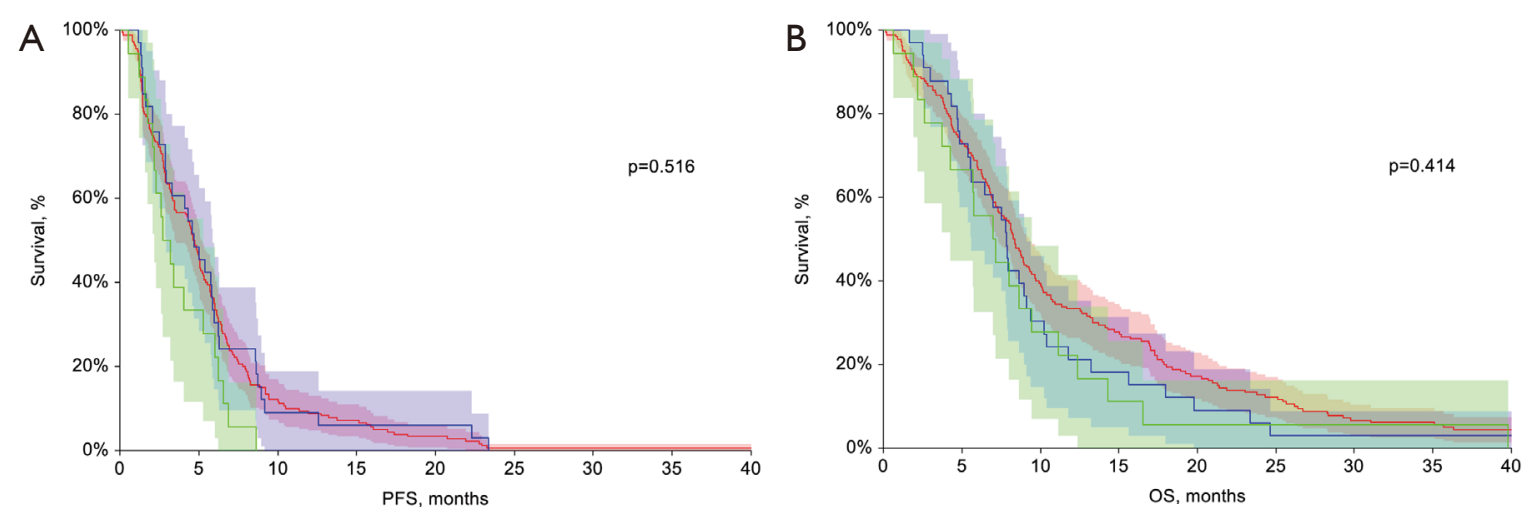

Figure S4 Prognostic impact of histologic type. Kaplan-Meier plots illustrating PFS and OS probability as a function of stage (A, B). Red line: Adenocarcinoma. Blue line: Squamous cell carcinoma. Green line: Other. The category 'Other' covers poorly differentiated nonsmall cell carcinoma and tumors with mixed histology treated as NSCLC. Colored areas represent $95 \%$ CIs. P-values for Log rank tests are shown.
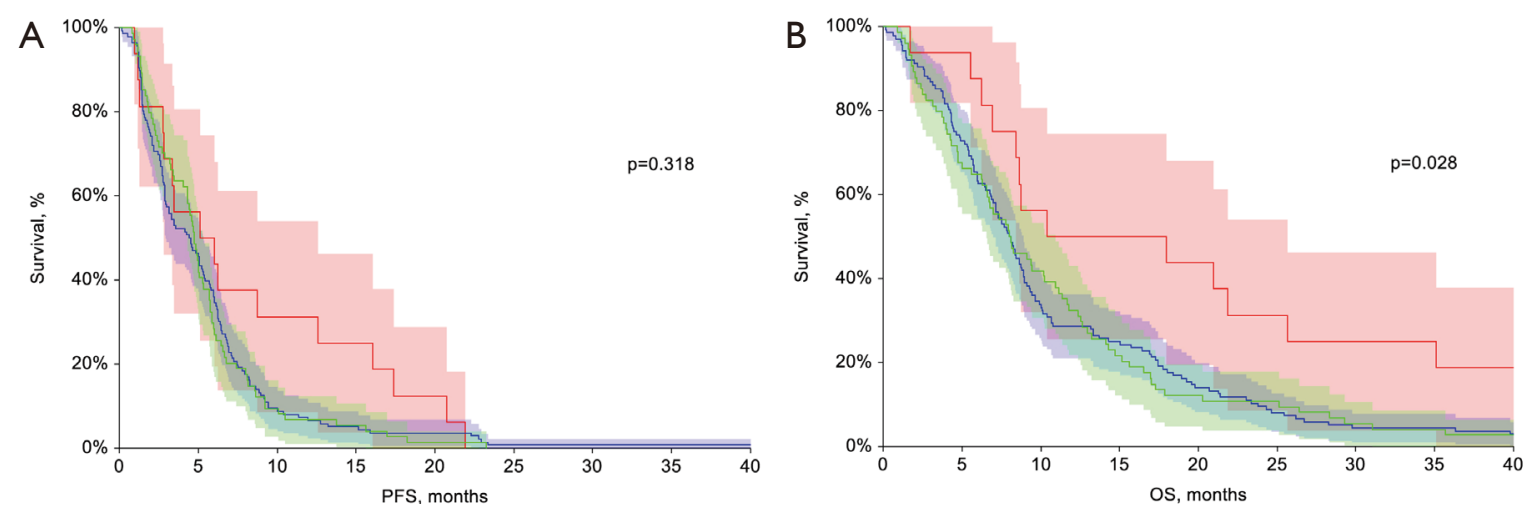

Figure S5 Prognostic impact of smoking status. Kaplan-Meier plots illustrating PFS and OS probability as a function of smoking status (A, B). Red line: Never smokers. Blue line: Previous smokers. Green line: Active smokers. Colored areas represent $95 \%$ CIs. P-values for Log rank tests are shown. 

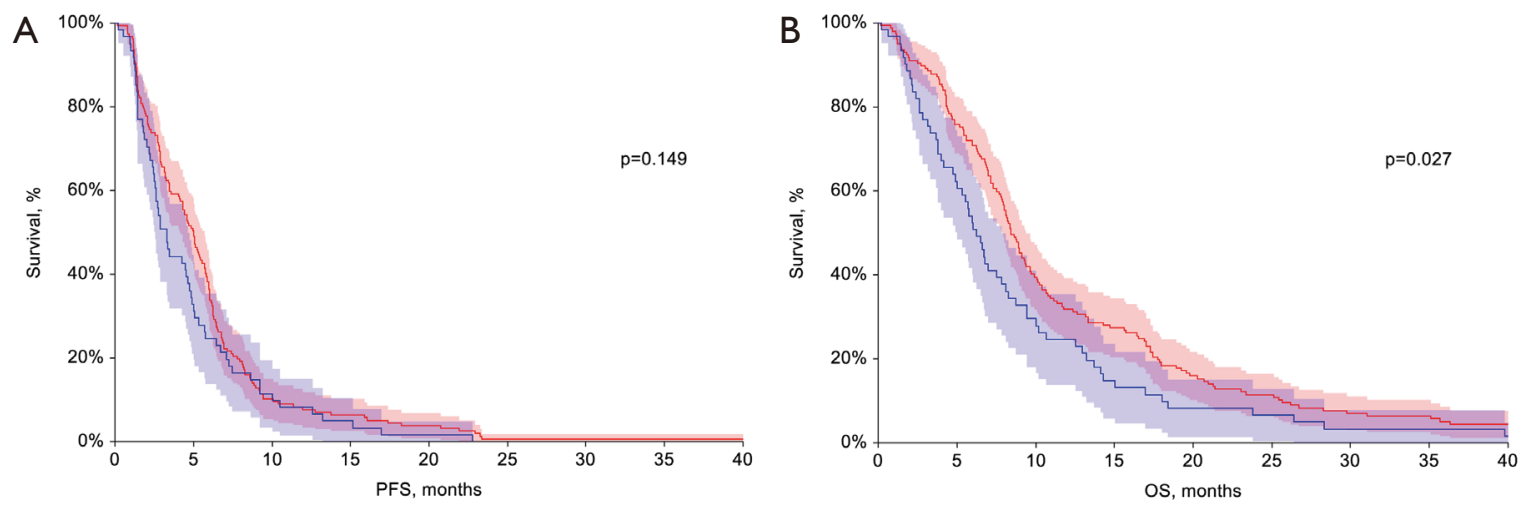

Figure S6 Prognostic impact of LDH status. Kaplan-Meier plots illustrating PFS and OS probability as a function of LDH status at baseline (A, B). Red line: $\mathrm{LDH}<250 \mathrm{U} / 1$. Blue line: $\mathrm{LDH} \geq 250 \mathrm{U} / 1$. Colored areas represent $95 \%$ CIs. P-values for Log rank tests are shown.

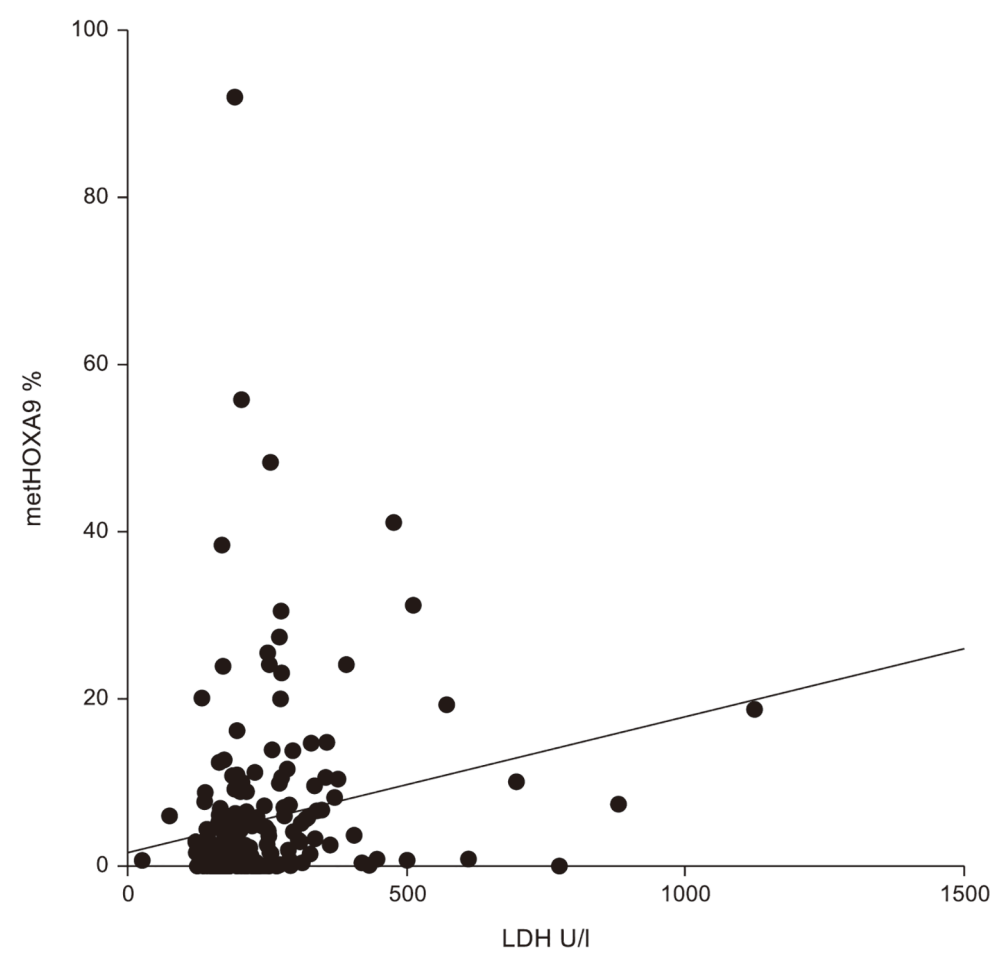

Figure S7 LDH in relation to metHOXA9. Scatter plot of LDH in U/l against metHOXA9 in percent showing a weak correlation depicted by a straight line, $r=0.31$ (A). 


\section{Multivariate analyses}

Table S1 Multiple Cox regression analysis, all covariates

\begin{tabular}{|c|c|c|c|c|}
\hline Covariate & $\mathrm{HR}$ & $\begin{array}{c}95 \% \mathrm{Cl} \\
\text { lower }\end{array}$ & $\begin{array}{c}95 \% \mathrm{Cl} \\
\text { upper }\end{array}$ & P-value \\
\hline \multicolumn{5}{|l|}{ metHOXA9 status, 1 cycle of treatment } \\
\hline Detectable & 3.619 & 2.085 & 6.280 & $<0.001^{*}$ \\
\hline \multicolumn{5}{|l|}{ Sex } \\
\hline Female (reference) & 1 & & & \\
\hline \multicolumn{5}{|l|}{ Smoking status } \\
\hline Never (reference) & 1 & & & \\
\hline Previous & 1.584 & 0.808 & 3.103 & 0.180 \\
\hline Active & 1.722 & 0.859 & 3.450 & 0.125 \\
\hline \multicolumn{5}{|l|}{ Histology } \\
\hline \multicolumn{5}{|l|}{ Treatment } \\
\hline Platinum and vinorelbine (reference) & 1 & & & \\
\hline Other & 0.637 & 0.400 & 1.014 & 0.057 \\
\hline \multicolumn{5}{|l|}{ Stage } \\
\hline Stage 3 & 1 & & & \\
\hline Stage 4 & 0.984 & 0.622 & 1.556 & 0.945 \\
\hline Age (numeric) & 1.001 & 0.983 & 1.020 & 0.886 \\
\hline LDH baseline (numeric) & 1.002 & 1.001 & 1.003 & $0.006^{*}$ \\
\hline Time-varying coefficient, metHOXA9 & 0.998 & 0.997 & 0.999 & $0.003^{*}$ \\
\hline
\end{tabular}

Multiple Cox regression analysis performed on $n=192$ patients and 189 events with complete data for all covariates. Table presents hazard ratios $(\mathrm{HR}), 95 \% \mathrm{Cls}$, and $\mathrm{p}$-values. *Statistically significant covariates.

The Cox regression model as reported in the manuscript (Table 2) was developed by the backward selection method. The full model (Table S1) was narrowed down by taking out one covariate with a high p-value and comparing the larger model with the smaller (nested) model by the likelihood-ratio test. If there was no significant difference between the two models, i.e. the missing covariate did not contribute significantly to the model, the smaller model was kept for further model development. There was no interaction between LDH and metHOXA9 neither as continuous nor binary variables.

The time varying coefficient (metHOXA9 status interacting with time) was included from the beginning. We tested the full model as described above (Table S1) but without the time varying coefficient with the proportional hazards test, and metHOXA9 status violated the proportional hazards assumption. The solution was to include a time varying coefficient in the model.

Missing data were treated as such and not included in the model or in other statistical analyses. 